\begin{tabular}{|c|c|}
\hline \multirow{3}{*}{ 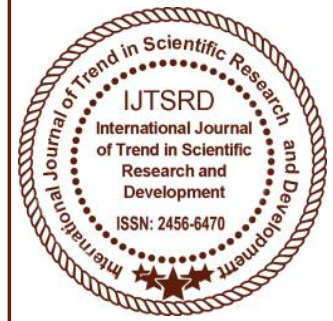 } & $\begin{array}{l}\text { International Journal of Trend in Scientific } \\
\text { Research and Development (IJTSRD) }\end{array}$ \\
\hline & UGC Approved International Open Access Journal \\
\hline & ISSN No: 2456 - 6470 | www.ijtsrd.com | Volume - 1 | Issue - 5 \\
\hline
\end{tabular}

\title{
A New Hendecagonal Fuzzy Number for Optimization Problems
}

\author{
M. Revathi \\ Assistant professor, \\ Department of Mathematics, Tamilnadu College of \\ Engineering,Coimbatore,Tamilnadu, India \\ R. Saravanan \\ Assistant professor, \\ Department of Mathematics, Tamilnadu College of \\ Engineering,Coimbatore,Tamilnadu, India
}

\section{ABSTRACT}

A new fuzzy number called Hendecagonal fuzzy number and its membership function is introduced, which is used to represent the uncertainty with eleven points. The fuzzy numbers with ten ordinates exists in literature. The aim of this paper is to define Hendecagonal fuzzy number and its arithmetic operations. Also a direct approach is proposed to solve fuzzy assignment problem (FAP) and fuzzy travelling salesman (FTSP) in which the cost and distance are represented by Hendecagonal fuzzy numbers. Numerical example shows the effectiveness of the proposed method and the Hendecagonal fuzzy number.

Keywords: Hendecagonal fuzzy number, Alpha cut, Fuzzy arithmetic, Fuzzy Assignment problem, Fuzzy transportation problem.

\section{INTRODUCTION}

A fuzzy number is a quantity whose values are imprecise, rather than exact as in the case with singlevalued function. The generalization of real number is the main concept of fuzzy number. In real world applications all the parameters may not be known precisely due to uncontrollable factors.

L.A.Zadeh introduced fuzzy set theory in 1965 . Different types of fuzzy sets [17] are defined in order to clear the vagueness of the existing problems.
D.Dubois and H.Prade has defined fuzzy number as a fuzzy subset of real line [8]. In literature, many type of fuzzy numbers like triangular fuzzy number, trapezoidal fuzzy number, pentagonal fuzzy number, hexagonal fuzzy number, heptagonal fuzzy number, octagonal fuzzy number, nanagonal fuzzy number, decagonal fuzzy number have been introduced with its membership function. These membership functions got many applications and many operations were done using these fuzzy numbers [2], [12] [14], [15].

In much decision analysis, the uncertainty existing in input information is usually represented as fuzzy numbers [1],. S.H.Chen introduced maximization and minimization of fuzzy set, uncertainty and information [4]. The arithmetic operations, alpha cut and ranking function are already introduced for existing fuzzy numbers by C.B.Chen and C.M.Klein,T.S.Liou and M.J.Wang [6],[11]. When the vagueness arises in eleven different points it is difficult to represent the fuzzy number. In this paper a new type of fuzzy number named as hendecagonal fuzzy number is defined with its membership function. The arithmetic operations, alpha cut and ranking procedure for hendecagonal fuzzy numbers are introduced to solve fuzzy assignment problem (FAP) and fuzzy travelling salesman problem (FTSP).In literature, many methods were proposed for fuzzy optimization with uncertain parameters $[3],[5],[10],[13]$. Here uncertainty in assignment cost 
International Journal of Trend in Scientific Research and Development (IJTSRD) ISSN: 2456-6470

and travelling distance are represented by new fuzzy number named as Hendecagonal fuzzy number, which are ranked using the ranking function introduced by R.R.Yager [16],[7]. Numerical examples show the effectiveness of the proposed method and the new fuzzy number, It is simple and very easy to understand and can be applied in many real life problems.

\section{Preliminary}

Definition 2.1: The membership grade corresponds to the degree to which an element is compatible with the concept represented by fuzzy set.

Definition 2.2: Let $X$ denote a universal set. Then the characteristic function which assigns certain values or a membership grade to the elements of this universal set within a specified range $[0,1]$ is known as membership function $\&$ the set thus defined is called a fuzzy set.

Definition 2.3: Let $X$ denote a universal set. Then the membership function $\mu_{A}$ by using a fuzzy set $\mathrm{A}$ is usually denoted as $\mu_{A}: X \rightarrow I$, where $I=[0,1]$

Definition 2.4: An $\alpha$-cut of a fuzzy set $\mathrm{A}$ is a crispest $A^{\alpha}$ that contains all the elements of the universal set $\mathrm{X}$ that have a membership grade in A greater or equal to specified value of $\alpha$ Thus

$$
A^{\alpha}=\left\{x \in X, \mu_{A}(x) \geq \alpha\right\}, 0 \leq \alpha \leq 1
$$

Definition 2.5: A fuzzy set $\tilde{\mathrm{A}}$ is a convex fuzzy set if and only if each of its $\alpha-$ cuts $A^{\alpha}$ is a convex set.

Definition2.6: A fuzzy set $\tilde{A}$ is a fuzzy number if (i) For all $\alpha \in(0,1]$ the $\alpha$-cut sets $A_{\alpha}$ is a convex set (ii) $\mu_{\tilde{A}}$ is an upper semi continuous function.

Definition 2.7: A triangular membership function is specified by three parameters $[a, b, c]$ as follows

$$
\mu(\mathrm{x}: \mathrm{a}, \mathrm{b}, \mathrm{c})= \begin{cases}(x-a) /(b-a) & , a \leq x \leq b \\ 1 & , x=b \\ (c-x) /(c-b) & , b \leq x \leq c \\ 0 & , \text { otherwise }\end{cases}
$$

This function is determined by the choice of the parameter $\mathrm{a}, \mathrm{b}, \mathrm{c}$ where $x_{i j} \in[0,1]$

Definition 2.8: A trapezoidal fuzzy number $\tilde{A}=(a, b, c, d)$ is a fuzzy number with membership function of the form

$$
\mu(x: a, b, c, d)=\left\{\begin{array}{cc}
(x-a) /(b-a) & , a \leq x \leq b \\
1 & , \quad b \leq x \leq c \\
(d-x) /(d-c) & , \quad c \leq x \leq d \\
0 & , \quad \text { otherwise }
\end{array}\right.
$$

\section{HENDECAGONAL FUZZY NUMBERS}

Definition 3.1: The parametric form of Hendecagonal Fuzzy Number is defined as $\tilde{U}=\left(P_{1}(r), Q_{1}(s), R_{1}(t), S_{1}(u), T_{1}(v), P_{2}(r), Q_{2}(s), R_{2}(t), S_{2}(u), T_{2}(v)\right) \quad, \quad$ for $r \in[0,0.2] s \in[0.2,0.4] t \in[0.4,0.6] u \in[0.6,0.8]$ and $v \in[0.8,1]$ where $P_{1}(r), Q_{1}(s), R_{1}(t), S_{1}(u)$ and $T_{1}(v)$ are bounded left continuous non decreasing functions over [0,0.2], [0.2,0.4], [0.4,0.6], [0.6,0.8] and [0.8.1],

$P_{2}(r), Q_{2}(s), R_{2}(t), S_{2}(u)$ and $T_{2}(v)$ Are bounded left continuous non increasing functions over $[0,0.2],[0.2,0.4],[0.4,0.6],[0.6,0.8]$ and $[0.8 .1]$.

\section{Definition 3.2: A fuzzy number} $A=\left(a_{1}, a_{2}, a_{3}, a_{4}, a_{5}, a_{6}, a_{7}, a_{8}, a_{9}, a_{10}, a_{11}\right)$ is said to be a Hendecagonal fuzzy number if its membership function is given by

$$
\begin{aligned}
& \frac{1}{5}\left(\frac{x-a_{1}}{a_{2}-a_{1}}\right) \quad, a_{1} \leq x \leq a_{2} \\
& \frac{1}{5}+\frac{1}{5}\left(\frac{x-a_{2}}{a_{3}-a_{2}}\right), a_{2} \leq x \leq a_{3} \\
& \frac{2}{5}+\frac{1}{5}\left(\frac{x-a_{3}}{a_{4}-a_{3}}\right), a_{3} \leq x \leq a_{4} \\
& \frac{3}{5}+\frac{1}{5}\left(\frac{x-a_{4}}{a_{5}-a_{4}}\right), a_{4} \leq x \leq a_{5} \\
& \frac{4}{5}+\frac{1}{5}\left(\frac{x-a_{5}}{a_{6}-a_{5}}\right), a_{5} \leq x \leq a_{6} \\
& \mu_{U}(x)=\left\{1-\frac{1}{5}\left(\frac{x-a_{6}}{a_{7}-a_{6}}\right), a_{6} \leq x \leq a_{7}\right. \\
& \frac{4}{5}-\frac{1}{5}\left(\frac{x-a_{7}}{a_{8}-a_{7}}\right), a_{7} \leq x \leq a_{8} \\
& \frac{3}{5}-\frac{1}{5}\left(\frac{x-a_{8}}{a_{9}-a_{8}}\right), a_{8} \leq x \leq a_{9} \\
& \frac{2}{5}-\frac{1}{5}\left(\frac{x-a_{9}}{a_{10}-a_{9}}\right), a_{9} \leq x \leq a_{10} \\
& \frac{1}{5}\left(\frac{a_{11}-x}{a_{11}-a_{10}}\right) \quad, a_{10} \leq x \leq a_{11} \\
& 0 \\
& \text {,Otherwise }
\end{aligned}
$$

Figure 1 shows the graphical representation of Hendecagonal fuzzy number. 
Hendecagonal fuzzy number

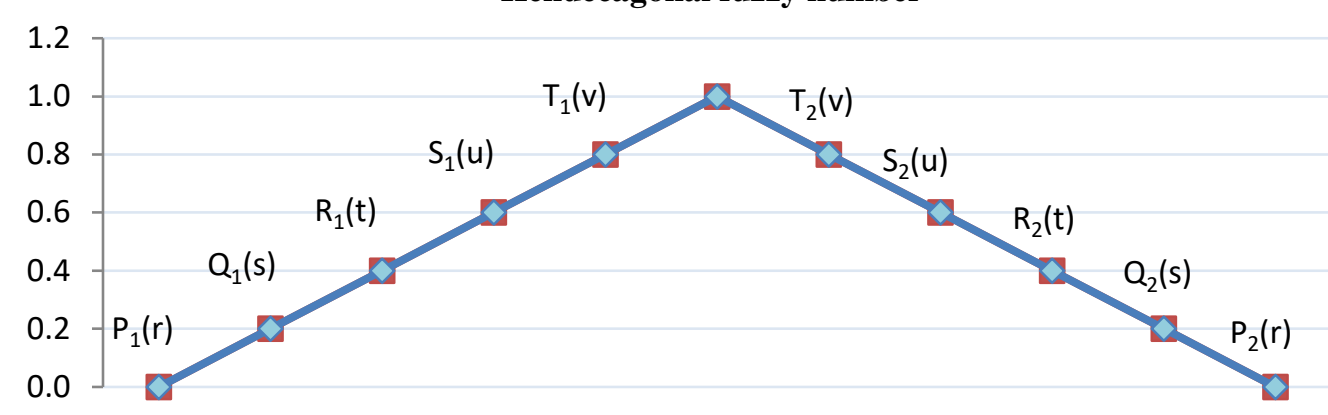

Figure 1: Graphical representation of Hendecagonal fuzzy number

\section{ARITHMETIC OPERATIONS HENDECAGONAL FUZZY NUMBERS}

In this section, arithmetic operations between two Hendecagonal fuzzy numbers, defined on universal set of real numbers $\mathrm{R}$, are presented.

Let $A=\left(a_{1}, a_{2}, a_{3}, a_{4}, a_{5}, a_{6}, a_{7}, a_{8}, a_{9}, a_{10}, a_{11}\right) \quad$ and $B=\left(b_{1}, b_{2}, b_{3}, b_{4}, b_{5}, b_{6}, b_{7}, b_{8}, b_{9}, b_{10}, b_{11}\right) \quad$ be two hendecagonal fuzzy number then

(i) Addition of two hendecagonal fuzzy numbers

$$
\begin{gathered}
\tilde{A} \oplus \tilde{B}=\left(c_{1}, c_{2}, c_{3}, c_{4}, c_{5}, c_{6}, c_{7}, c_{8}, c_{9}, c_{10}, c_{11}\right) \\
=\left(a_{1}+b_{1}, a_{2}+b_{2}, a_{3}+b_{3}, a_{4}+b_{4}, a_{5}+b_{5}, a_{6}+b_{6},\right. \\
\left.a_{7}+b_{7}, a_{8}+b_{8}, a_{9}+b_{9}, a_{10}+b_{10}, a_{11}+b_{11}\right)
\end{gathered}
$$

\section{(ii) Scalar multiplication of hendecagonal fuzzy}

\section{numbers}

$$
\lambda \tilde{A}=\left\{\begin{array}{l}
\left(\lambda a_{1}, \lambda a_{2}, \lambda a_{3}, \lambda a_{4}, \lambda a_{5}, \lambda a_{6}, \lambda a_{7}, \lambda a_{8}, \lambda a_{9}, \lambda a_{10}, \lambda a_{11}\right) \text { if } \lambda>0 \\
\left(\lambda a_{11}, \lambda a_{10}, \lambda a_{9}, \lambda a_{8}, \lambda a_{7}, \lambda a_{6}, \lambda a_{5}, \lambda a_{4}, \lambda a_{3}, \lambda a_{2}, \lambda a_{1}\right) \text { if } \lambda<0
\end{array}\right.
$$

\section{(iii) Subtraction of two hendecagonal fuzzy}

\section{numbers}

$$
\begin{aligned}
& \tilde{A} \Theta \tilde{B}=\tilde{A} \oplus(-\tilde{B}) \\
& =\left(a_{1}-b_{11}, a_{2}-b_{10}, a_{3}-b_{9}, a_{4}-b_{8}, a_{5}-b_{7}, a_{6}-b_{6},\right. \\
& \left.\quad a_{7}-b_{5}, a_{8}-b_{4}, a_{9}-b_{3}, a_{10}-b_{2}, a_{11}-b_{1}\right)
\end{aligned}
$$

\section{RANKING HENDECAgONAL FUZZY NUMBERS}

The ranking method proposed in [4] is used to rank the hendecagonal fuzzy numbers.
ON The ranking function $r: F(R) \rightarrow R$ where $\mathrm{F}(\mathrm{R})$ is a set of fuzzy number defined on set of real numbers, which maps each fuzzy number into the real line, where the natural order exists, i.e.

(i) $\tilde{A}>\widetilde{B}$ iff $r(\tilde{A})>r(\widetilde{B})$

(ii) $\tilde{A}<\widetilde{B}$ iff $r(\tilde{A})<r(\widetilde{B})$

(iii) $\tilde{A}=\widetilde{B}$ iff $r(\tilde{A})=r(\widetilde{B})$

Let $\quad A=\left(a_{1}, a_{2}, a_{3}, a_{4}, a_{5}, a_{6}, a_{7}, a_{8}, a_{9}, a_{10}, a_{11}\right) \quad$ and $B=\left(b_{1}, b_{2}, b_{3}, b_{4}, b_{5}, b_{6}, b_{7}, b_{8}, b_{9}, b_{10}, b_{11}\right)$

be two hendecagonal fuzzy numbers then

$r(\tilde{A})=\frac{a_{1}+a_{2}+a_{3}+a_{4}+a_{5}+a_{6}+a_{7}+a_{8}+a_{9}+a_{10}+a_{11}}{11}$

and $r(\widetilde{B})=\frac{b_{1}+b_{2}+b_{3}+b_{4}+b_{5}+b_{6}+b_{7}+b_{8}+b_{9}+b_{10}+b_{11}}{11}$

\section{Fuzzy Assignment Problem AND fuzzy Travelling Salesman Problem}

In this section, mathematical formulation of fuzzy assignment problem is given and a direct approach is proposed to solve FAP and FTSP. The method is applicable for all optimization problems.

\section{A. Formulation of Fuzzy Assignment Problem}

Let there be $m$ Tasks and $m$ Workers, $\widetilde{\mathrm{C}}_{\mathrm{ij}}$ be the cost of assigning $\mathrm{i}^{\text {th }}$ Worker to the $\mathrm{j}^{\text {th }}$ Task and the uncertainty in cost is here represented as Hendecagonal fuzzy numbers. Let $\mathrm{x}_{\mathrm{ij}}$ be the decision variable define 
$x_{i j}=\left\{\begin{array}{l}1 \text { if the } \mathrm{i}^{\text {th }} \text { person is assigned to the } \mathrm{j}^{\text {th }} \text { job } \\ 0 \text { otherwise }\end{array}\right.$

Then the fuzzy assignment problem can be mathematically stated as follows

Minimize $\tilde{Z}=\sum_{i=1}^{m} \sum_{j=1}^{m} \tilde{C}_{i j} x_{i j}$

Subject to $\sum_{j=1}^{m} x_{i j}=1, i=1,2 \ldots m$;

$\sum_{i=1}^{m} x_{i j}=1, j=1,2 \ldots m$

\section{B. Formulation of Fuzzy Travelling Salesman Problem}

The travelling salesman problem deals with finding shortest path in a n-city where each city is visited exactly once. The travelling salesman problem is similar to assignment problem that excludes sub paths. Specifically in an n-city situation define

$x_{i j}=\left\{\begin{array}{l}1, \text { if city } j \text { is reached from city } i \\ 0, \text { otherwise }\end{array}\right.$

Here $\tilde{d}_{i j}$ is the distance from city $\mathrm{i}$ to city $\mathrm{j}$ which is Hendecagonal fuzzy number. Mathematically FTSP can be stated as

$\operatorname{Minimize} \quad \tilde{z}=\sum_{i=1}^{n} \sum_{j=1}^{n} \tilde{d}_{i j} x_{i j}, \quad \tilde{d}_{i j}=\infty$ for all $i=j$

Subject to $\sum_{j=1}^{n} x_{i j}=1, i=1,2 \ldots n$

$$
\sum_{i=1}^{n} x_{i j}=1, j=1,2 \ldots n \quad, x_{i j} \in[0,1]
$$

\section{NUMERICAL EXAMPLE}

In this section numerical examples are given to illustrate the proposed method and it is shown that the proposed method offers an effective way for handling FAP as well as FTSP.

Example 7.1: A manufacturing company manufactures a certain type of spare parts with three different machines. The company official has to execute three jobs with three machines. The information about the cost of assignment is imprecise and here Hendecagonal Fuzzy numbers are used to represent the cost. The fuzzy assignment problem is given in Table 1.

\section{Solution:}

Step 1: Calculate the ranking value of each fuzzy cost is given in Table 2.

Step 2: Encircle the fuzzy cost with least ranking value in each row and examine all the encircled fuzzy costs and identify the encircled fuzzy cost that is uniquely encircled in both row wise and column wise. Assign it and delete the corresponding row and column. The resultant table is given in Table 3.

Step 3:If the cost is not uniquely selected both row wise and column wise then choose next minimum and proceed as in step 2.This process is continued until the fuzzy cost is uniquely selected row and column wise.

Then the optimal assignment is $J_{1} \rightarrow M_{1}, J_{2} \rightarrow M_{2}$, $J_{3} \rightarrow M_{3}$ with the optimal assignment cost $(4,8,12,16,21,29,35,39,43,47,54)$ and its crisp value is $\mathrm{r}(\mathrm{C})=28$

Table 1: Fuzzy Assignment Problem with Hendecagonal Fuzzy Cost

\begin{tabular}{|c|c|c|c|c|}
\hline \multirow{2}{*}{} & \multicolumn{3}{|c|}{ Machines } & M3 \\
\cline { 3 - 5 } & J1 & $(1,3,5,7,9,11,13,15,17,19,21)$ & $(2,4,6,8,10,12,14,16,18,20,22)$ & $(1,2,3,4,5,6,7,8,9,10,11)$ \\
\hline \multirow{2}{*}{ 으 } & J2 & $(3,7,11,13,17,21,22,25.29,32,40)$ & $(2,4,6,8,9,13,15,16,18,20,21)$ & $(2,3,7,8,9,11,13,15,16,21,33)$ \\
\cline { 2 - 5 } & & & & \\
\cline { 2 - 5 } & J3 & $(1,2,3,4,7,10,13,15,16,17,22)$ & $(5,8,10,13,16,21,23,28,31,32)$ & $(4,6,7,9,10,11,18,23,24,26,27)$ \\
\hline
\end{tabular}




\section{Table 2: Ranking value of Hendecagonal Fuzzy cost}

\begin{tabular}{|c|c|c|c|c|}
\hline & \multicolumn{3}{|c|}{ Machines } \\
\hline & & M1 & M2 & M3 \\
\hline \multirow{3}{*}{$\stackrel{\infty}{0}$} & J1 & $r\left(\tilde{c}_{11}\right)=11$ & $r\left(\tilde{c}_{12}\right)=12$ & $r\left(\tilde{c}_{13}\right)=6$ \\
\hline & $\mathrm{J} 2$ & $r\left(\tilde{c}_{21}\right)=20$ & $r\left(\tilde{c}_{22}\right)=12$ & $r\left(\tilde{c}_{23}\right)=13$ \\
\hline & $\mathrm{J} 3$ & $r\left(\tilde{c}_{31}\right)=10$ & $r\left(\tilde{c}_{32}\right)=19$ & $r\left(\tilde{c}_{33}\right)=15$ \\
\hline
\end{tabular}

\begin{tabular}{|c|c|c|c|c|}
\hline \multicolumn{5}{|c|}{ Table 3: Encircled Fuzzy Cost } \\
\cline { 3 - 5 } \multicolumn{2}{|c|}{} & M1 & Machines & M3 \\
\hline \multirow{3}{*}{$\approx 0$} & J1 & $(1,3,5,7,9,11,13,15,17,19,21)$ & $(2,4,6,8,10,12,14,16,18,20,22)$ & $(1,2,3,4,5,6,7,8,9,10,11)$ \\
\cline { 2 - 5 } & J2 & $(3,7,11,13,17,21,22,25.29,32,40)$ & $(2,4,6,8,9,13,15,16,18,20,21)$ & $(2,3,7,8,9,11,13,15,16,21,33)$ \\
\cline { 2 - 5 } & J3 & $(1,2,3,4,7,10,13,15,16,17,22)$ & $(5,8,10,13,16,21,23,28,31,32)$ & $(4,6,7,9,10,11,18,23,24,26,27)$ \\
\hline
\end{tabular}

Example 7.2:Let us consider a fuzzy travelling salesman problem with three cities $\mathrm{C}_{1}, \mathrm{C}_{2}, \mathrm{C}_{3}$. The distance matrix $\left\lfloor\tilde{d}_{i j}\right\rfloor$ is given whose elements are Hendecagonal fuzzy numbers. A salesman must travel from city to city to maintain his accounts. The problem is to find the optimal assignment, so that the assignment minimize the total distance of visiting all cities and return to starting city. The fuzzy travelling salesman problem is given in Table 4.

\section{Solution:}

Step 1: Calculate the ranking value of each fuzzy distance is given in Table 5.

Step 2: Encircle the fuzzy distance with least ranking value in each row and examine all the encircled fuzzy distance to find the uniquely encircled fuzzy distance in both row wise and column wise. Assign it and delete the corresponding row and column. The resultant table is given in Table 6.

Step 3:If the distance is not uniquely selected both row wise and column wise then choose next minimum and repeat the step 2.This process is continued until the fuzzy distance is uniquely selected row and column wise.

Thus the optimal assignment is $\mathrm{C}_{1} \rightarrow \mathrm{C}_{2}$, $C_{2} \rightarrow C_{3}, C_{3} \rightarrow C_{1}$ with the optimal distance $(5,9,16,20,26,33,40,46,50,58,77)$ and its crisp value is $r\left(\tilde{d}_{i j}\right)=34.55$ 
International Journal of Trend in Scientific Research and Development (IJTSRD) ISSN: 2456-6470

Table 4: Fuzzy Travelling Salesman Problem with Hendecagonal Fuzzy Distance

\begin{tabular}{|c|c|c|c|c|}
\hline & \multicolumn{3}{|c|}{ CITY } \\
\hline & & 'C1 & $\mathrm{C2}$ & C3 \\
\hline \multirow{3}{*}{$\vec{\sigma}$} & $\mathrm{C} 1$ & $\infty$ & $(2,4,6,8,10,12,14,16,18,20,22)$ & $(1,2,3,4,5,6,7,8,9,10,11)$ \\
\hline & $\mathrm{C} 2$ & $(3,7,11,13,17,21,22,25.29,32,40)$ & $\infty$ & $(2,3,7,8,9,11,13,15,16,21,33)$ \\
\hline & C3 & $(1,2,3,4,7,10,13,15,16,17,22)$ & $(5,8,10,13,16,21,23,28,31,32)$ & $\infty$ \\
\hline
\end{tabular}

Table 5: Ranking value of Hendecagonal Fuzzy Distance

\begin{tabular}{|c|c|c|c|c|}
\hline & \multicolumn{3}{|c|}{ CITY } \\
\hline & & $\mathrm{C} 1$ & ${ }^{\mathrm{C}} 1$ & $\mathrm{C} 1$ \\
\hline \multirow{3}{*}{$\vec{v}$} & $\mathrm{C} 1$ & $\infty$ & $r\left(\tilde{c}_{12}\right)=12$ & $r\left(\tilde{c}_{13}\right)=6$ \\
\hline & $\mathrm{C} 2$ & $r\left(\tilde{c}_{21}\right)=20$ & $\infty$ & $r\left(\tilde{c}_{23}\right)=13$ \\
\hline & C3 & $r\left(\tilde{c}_{31}\right)=10$ & $r\left(\tilde{c}_{32}\right)=19$ & $\infty$ \\
\hline
\end{tabular}

Table 3: Encircled Fuzzy Distance

\begin{tabular}{|c|c|c|c|c|}
\hline & \multicolumn{3}{|c|}{ CITY } \\
\hline & & $\mathrm{C} 1$ & $\mathrm{C} 1$ & M3 \\
\hline \multirow{3}{*}{$\underline{Z}$} & $\mathrm{C} 1$ & $\infty$ & $(2,4,6,8,10,12,14,16,18,20,22)$ & $(1,2,3,4,5,6,7,8,9,10,11)$ \\
\hline & $\mathrm{C} 2$ & $\begin{array}{c}(3,7,11,13,17,21,22,25.29,32, \\
40)\end{array}$ & $\infty$ & $(2,3,7,8,9,11,13,15,16,21,33)$ \\
\hline & $\mathrm{C} 3$ & $(1,2,3,4,7,10,13,15,16,17,22)$ & $(5,8,10,13,16,21,23,28,31,32)$ & $\infty$ \\
\hline
\end{tabular}

\section{CONCLUSION AND FUTURE ENHANCEMENT}

In this paper, a new fuzzy number is developed for solving optimization problem with Hendecagonal fuzzy cost and fuzzy distance. The optimal solution to FAP and FTSP obtained by the proposed method is same as that of the optimal solution obtained by the existing methods. However the proposed method is simpler, easy to understand and it takes few steps for obtaining the fuzzy optimal solution. Numerical example shows that the proposed method offers an effective tool for handling the fuzzy assignment problem. In future, the generalization of Hendecagonal fuzzy number is developed to solve optimization problems. 
International Journal of Trend in Scientific Research and Development (IJTSRD) ISSN: 2456-6470

\section{REFERENCES}

[1] Amit Kumar and Anil Gupta, "Assignment and Travelling salesman problems with coefficient as LR fuzzy parameter", International Journal of applied science and engineering 10(3), 155-170, 2010.

[2] J.J.Buckly, "Possibility linear programming with triangular fuzzy numbers", Fuzzy sets and systems, 26, 135-138, 1988.

[3] A.Chaudhuri,K.De,"Fuzzy multi-objective linear programming for Travelling salesman problem", Afr. J. Math. Comp. Sci. Res., 4(2), 64-70, 2011.

[4] S.H.Chen, "Ranking fuzzy numbers with maximizing set and minimizing set and systems", 17, 113-129, 1985.

[5] M.S.Chen, "On a fuzzy assignment problem”, Tamkang Journal, 22, 407-411, 1985.

[6] C.B.Chen and C.M.Klein, "A simple approach to ranking a group of aggregated fuzzy utilities", IEEE Trans syst., 27, 26-35, 1997.

[7] F.Choobinesh and H.Li, "An index for ordering fuzzy numbers", Fuzzy sets and systems, 54,287-294, 1993.

[8] D.Doibus, H.Prade, "Fuzzy sets and systems theory and applications", Academic press, Newyork, 1980.

[9] S.Kikuchi, "A method to defuzzify the fuzzy numbers, Transportation problems application", Fuzzy sets and systems, 116,3-6, 2000.
[10] H.W.Kuhn, "The Hungarian method for the Assignment problem”, Naval Res.Logistics, 2, 83-97,1955.

[11] T.S.Liou, M.J.Wang, "Ranking fuzzy number with integral values", Fuzzy sets and system, 50, 247-255,1992.

[12] S.Mukherjee,K.Basu, "Application of fuzzy ranking method for solving Assignment problems with fuzzy costs", International Journal of Computational and Applied Mathematics, 5,359-368,2010.

[13] M.Revathi, R.Saravanan and K.Rathi, "A new approach to solve travelling salesman problem under fuzzy environment", International journal of current research,7(12), 2412824132, 2015.

[14] K. Rathi and S. Balamohan," Representation and ranking of fuzzy numberswith heptagonal membership function using value and ambiguity index", Applied Mathematical Sciences, 8(87), 4309-4321, 2014.

K. Rathi, S. Balamohan, P.Shanmugasundaram and M.Revathi," Fuzzy row penalty method to solve assignment problem with uncertain parameters", Global journal of Pure and Applied Mathematics, 11(1), 39-44, 2015.

[16] R.R.Yager, "A characterization of extension principle", Fuzzy sets and systems, 18, 205217, 1986.

[17] L.A.Zadeh, "Fuzzy sets,Information and Control", 8, 338-353, 1965. 